\title{
Robert Eric Frykenberg, Christianity in India. From Beginnings to the Present
}

8 cartes, 13 illustrations, bibliographie, glossaire, index

New York, Oxford University Press, 2010, 564 p.

\section{Catherine Clémentin-Ojha}

\section{(2) OpenEdition}

Journals

Édition électronique

URL : http://journals.openedition.org/assr/25481

DOI : $10.4000 /$ assr. 25481

ISSN : $1777-5825$

Éditeur

Éditions de l'EHESS

Édition imprimée

Date de publication : 30 décembre 2013

Pagination : 196

ISSN : 0335-5985

\section{Référence électronique}

Catherine Clémentin-Ojha, « Robert Eric Frykenberg, Christianity in India. From Beginnings to the Present », Archives de sciences sociales des religions [En ligne], 164 | 2013, mis en ligne le 21 février 2014, consulté le 21 septembre 2020. URL : http://journals.openedition.org/assr/25481 ; DOI : https:// doi.org/10.4000/assr.25481

Ce document a été généré automatiquement le 21 septembre 2020.

(c) Archives de sciences sociales des religions 


\section{Robert Eric Frykenberg, Christianity in India. From Beginnings to the Present}

8 cartes, 13 illustrations, bibliographie, glossaire, index

New York, Oxford University Press, 2010, 564 p.

Catherine Clémentin-Ojha

\section{RÉFÉRENCE}

Robert Eric Frykenberg, Christianity in India. From Beginnings to the Present, 8 cartes, 13 illustrations, bibliographie, glossaire, index, New York, Oxford University Press, $2010,564 \mathrm{p}$. 
1 Robert Eric Frykenberg, professeur émérite de l'Université du Wisconsin, historien spécialiste de l'Inde coloniale, a consacré sa carrière à l'étude de deux grands sujets: les politiques de réforme foncière conduites par les administrateurs britanniques et les processus d'évangélisation de la société indienne. Quoique ces deux sujets présentent un grand nombre de différences, ils sont l'un et l'autre traversés par le même constat: celui de l'inévitable adaptation aux conditions de l'Inde d'idées et de méthodes d'organisation à l'origine étrangères à son sol. Le spécialiste d'histoire économique et sociale de l'Inde a ainsi montré que les administrateurs britanniques n'avaient pas fondamentalement révolutionné le régime
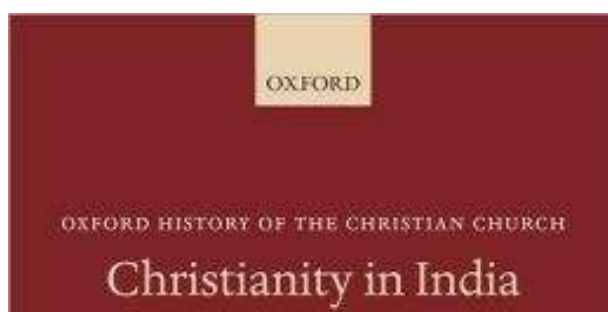

From Beginnings to the Present

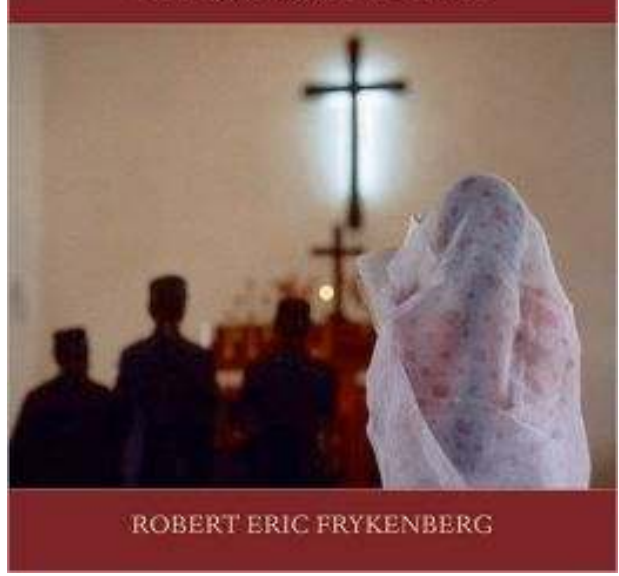
de propriété foncière. Ils avaient ainsi permis aux vieilles élites foncières de continuer à prospérer dans les nouvelles conditions économiques et sociales qu'ils avaient créées (Guntur District, 1788-1848: A History of Local Influence and Central Authority in South India, 1965).

C'est une continuité de même nature que l'historien des missions met en lumière dans ses études sur les chrétiens de l'Inde. Ainsi dans un ouvrage récent (voir mon compte rendu dans ASSR, 144), il montrait que le processus d'évangélisation ne devait être envisagé ni du seul point de vue des missionnaires - comme un transfert - ni du seul point de vue des populations évangélisées - comme une réception - mais comme le résultat de l'interaction continue entre missionnaires occidentaux et missionnaires indiens. Dans l'ouvrage présenté ici, l'auteur propose une réflexion d'ensemble sur la nature des changements sociaux qui ont surgi et ont été négociés dans les situations d'interactions interculturelles que l'évangélisation a générées en Inde. Suivant l'ordre chronologique, même s'il introduit des chapitres thématiques qui exigent de fréquents retours en arrière, il couvre deux mille ans d'histoire et un espace géographique non moins vaste, celui du sous-continent indien.

Quoiqu'il paraisse dans l'oxford History of the Christian Church, qui en vingt volumes entend couvrir l'histoire du christianisme sous toutes les latitudes, ce très ambitieux travail ne peut être considéré comme un manuel de référence sur le christianisme en Inde. Cela tient d'abord à ce que l'extraordinaire diversité des situations comme la richesse et la complexité du matériau ont obligé l'auteur à être très sélectif. Cela tient aussi aux propres convictions, religieuses et historiographiques, de l'auteur. Celles-ci ne sont pas non plus étrangères à ses choix et elles donnent souvent à son ouvrage un caractère militant. Frykenberg a pris le parti d'aborder les grandes questions à travers l'examen fouillé de situations particulières, qu'il traite comme autant d'exemples significatifs, en s'appuyant sur ses propres travaux ou en livrant une synthèse de ses nombreuses lectures. Pour tout ce dont il ne traite pas, il guide le lecteur vers des 
ouvrages plus spécialisés (la bibliographie est copieuse et n'omet pas les recherches les plus récentes), vers les volumes publiés par la Church History Association of India ou vers la History of Christianity in India en deux volumes de Stephen Neill, auteur qui a ouvert la voie à tous ceux qui comme Frykenberg sont soucieux de rendre compte du versant indien du christianisme en Inde, et non de son seul versant missionnaire comme l'ont fait longtemps les histoires classiques. Quoique restée inachevée (elle ne va pas au-delà de 1857, année charnière qui marque la fin de la East India Company et celle de l'Empire moghol), la somme de Neill reste un indispensable outil de travail en raison de sa vision synoptique. On ne trouve pas cette vue d'ensemble dans l'entreprise éditoriale de la Church History Association of India, dont les volumes sur les différentes communautés et Églises chrétiennes, au demeurant de très bonne qualité, ont été écrits par différents auteurs.

Parmi les choix de l'auteur est celui de traiter principalement de l'Inde du Sud. Cela se comprend puisque « presque toute l'histoire du christianisme [y] a pris place» (p. 169). La christianisation de l'Inde commence en effet avec l'installation sur la côte du sud-est d'une communauté de chrétiens qui se réclament de l'apôtre Thomas (intéressante discussion au chapitre 4 sur l'impossibilité de parvenir à quelque certitude sur la date de ces premiers chrétiens), et elle s'y poursuit plusieurs siècles plus tard quand, par un curieux hasard dont l'histoire abonde, le navigateur Vasco da Gama débarque sur cette même côte en 1498, donnant le point de départ de l'entreprise coloniale portugaise et des missions catholiques qui l'accompagnent. De nouvelles chrétientés voient alors le jour à Goa et au pays tamoul (très brève présentation des missions jésuites p. 137-140) tandis que la vieille communauté de saint-Thomas se divise, une partie passant sous la coupe de Rome, l'autre fondant une Église indépendante (dont on poursuivra l'histoire aux chapitres 9 et 12). Cela explique que, dans les décennies qui suivent, les chrétiens de Saint-Thomas sont confrontés à la puissante Église de Rome puis, en 1599, en partie soumis à sa juridiction. C'est toujours en Inde du Sud que, à partir du début du XVIII ${ }^{\mathrm{e}}$ siècle, les évangéliques piétistes puis, au XIX ${ }^{\mathrm{e}}$, les anglicans s'installent. Aujourd'hui encore l'Inde du Sud reste un terrain d'évangélisation de choix, même si désormais les missions pentecôtistes ont pris le pas sur les missions catholiques, évangéliques et anglicanes, et n'hésitent pas à attirer à elles leurs ouailles.

5 En fait, l'évangélisation reste impossible au Nord tant qu'y domine l'Empire moghol dont, autre coïncidence historique, la puissance s'est affirmée à peu près au moment même où les premiers Européens débarquaient en Inde du Sud. Elle ne commence à y gagner du terrain qu'à partir XIX siècle quand les Britanniques, qui depuis la fin du XVIII ${ }^{e}$ siècle ont évincé les Portugais et les autres puissances européennes et fortement ébranlé le pouvoir moghol, en prennent le contrôle politique. Des missions européennes et américaines s'implantent alors au Panjab, en Inde centrale, et au Bihar. Mais c'est surtout chez les groupes tribaux de l'Assam et du Nord-Est, que la conquête britannique a sortis de leur isolement géographique et culturel, qu'on observe un nombre significatif de conversions (la majorité de la population de ces régions est aujourd'hui chrétienne).

Plutôt que tenter de résumer cette gigantesque synthèse, qui elle-même condense une très riche matière, il me paraît plus intéressant de mettre en lumière sa principale thèse. Frykenberg l'énonce nettement dans sa conclusion : les chrétiens de l'Inde sont à tous égards indiens et partout leur religion a pris des traits indiens et porte le cachet des cultures nationales. Il faut donc voir leurs diverses communautés "comme de 
nombreux exemples de découvertes et d'appropriations indigènes du christianisme et non simplement comme des exemples de découverte et d'exploration chrétiennes des cultures autochtones (native) par des missionnaires ou par des savants étrangers à l'Inde. » (p. 454). L'ouvrage s'emploie à démontrer ce caractère indigène, à en exposer les traits saillants et à en tirer les conséquences. Retenons les principaux points de l'argumentation avant de nous interroger pour conclure sur le sens de cette démarche. Le premier est d'ordre sociologique. Si le christianisme en Inde est éminemment indien, c'est parce qu'il a été entièrement façonné par ces deux modes d'organisation sociale que l'on rencontre en Inde, le système des castes (jāti, litt. " naissance ») d'une part, le système des tribus (ādivāsī - et non adivāsi comme l'écrit l'auteur - "les habitants originaires ») d'autre part. Les traits spécifiques de ces deux systèmes (longuement expliqués aux chapitres 2 et 3 ) doivent être gardés à l'esprit si l'on veut comprendre la sociologie du christianisme en Inde. Si l'on veut comprendre plus particulièrement le fait que la plupart des chrétiens appartiennent à des castes inférieures ou à des groupes tribaux qui n'avaient été ni hindouisées ni islamisées. Le mode d'organisation sociale spécifique du système des castes, qui classe, hiérarchise et exclut les êtres humains selon qu'ils sont nés dans tel ou tel groupe, ainsi que les conceptions hindoues de la pureté sont en effet directement responsables du nombre infime de conversions parmi les castes supérieures. La majorité des chrétiens viennent de castes "intouchables", c'est-à-dire de groupes ostracisés parce que situés à l'extérieur des quatre classes (varna ) de la société brahmanique - que dans son souci de faire une présentation «indocentric » (p. 6) Frykenberg choisit de désigner par le terme savant sanskrit avarṇa [sans varna] (écrit āvarna dans tout l'ouvrage). Le grand problème de l'auteur, après avoir été celui d'autres historiens du christianisme indien, est d'expliquer l'échec de l'évangélisation auprès des castes supérieures, ou, ce qui revient au même, les raisons de son succès auprès des castes inférieures et des tribaux. S'agissant de ces derniers, leur conversion au christianisme tiendrait selon Frykenberg à ce que leur religion (dite " primal religion ») était en affinité avec la foi chrétienne et leur permit d'adopter cette dernière sans perdre leur identité culturelle (p. 10,13).

Le deuxième point de l'argumentation est d'ordre missiologique. Si le christianisme est éminemment indien, ce n'est pas seulement qu'il s'est adapté à la société indienne, c'est aussi que les Indiens eux-mêmes ont joué un rôle déterminant dans sa propagation. L'auteur ne démontre nulle part mieux ce point que dans le chapitre 6 consacré à la mission fondée au début du XvIII ${ }^{e}$ siècle à Tranquebar (pays tamoul), en décrivant les missionnaires du courant piétiste évangélique (de Halle) et leurs collaborateurs indiens comme des "go-betweens" (dubashis, littéralement «qui a deux langues») et l'évangélisation comme un processus d'appropriation mutuelle des deux cultures. Aussi n'y traite-t-il pas seulement de la carrière de Ziegenbalg et de ses successeurs (Schultze, Fabricius, Schwartz), comme on pouvait s'y attendre, mais aussi de celle des disciples indiens de Schwartz, ce qui est à relever car ils sont peu connus en dehors des cercles de spécialistes: Nellaiyan Vedayakam Sastriar, Sathyanathan Pillai, Sundaranandam David, Rasa Clorinda. Ce sont ces derniers qui pour une large part ont suscité les conversions, et non les missionnaires étrangers qui les avaient formés, parce que «les chrétiens de l'Inde, en fin de compte et par consensus, ne pouvaient être autre chose que profondément indiens» (p.168). Frykenberg offre d'autres exemples d'évangélisation en dehors du contrôle direct des missionnaires étrangers. 
9 Mais pour démontrer le caractère indien du christianisme en Inde, il ne suffit pas de montrer qu'il ne saurait être réduit à la saga missionnaire, il faut encore dire qu'il ne peut être davantage assimilé à la colonisation en faisant quelques rappels historiques. À cela il y a déjà une raison indéniable : la communauté des chrétiens de Saint-Thomas était installée en Inde dès les premiers siècles de notre ère, sinon dès le tout premier siècle (comme en sont convaincus les intéressés qui attribuent la conversion de leurs ancêtres à l'apôtre lui-même). Cela signifie, et là est l'argument décisif pour la démonstration recherchée, que ce ne sont pas les Européens qui ont apporté le christianisme en Inde. Autre argument: même une fois bien présents sur le terrain indien, les missionnaires ne furent pas des agents de la colonisation, le soutien des autorités coloniales, qui contrôlaient les territoires sur lesquels ils œuvraient, ne fut jamais inconditionnel. Au demeurant, à partir du XIX siècle, la puissance coloniale n'aurait pu asseoir son pouvoir ni le maintenir sans la collaboration des Indiens, ni sans faire de larges concessions aux hindous, qui forment la vaste majorité de la population : le Raj britannique lui-même fut "une entité hybride», "autant indienne que britannique » (p. 204), et il ne fut pas seulement indien, mais hindou, assure l'auteur. (p. 205)

10 En s'attachant à démontrer que l'histoire du christianisme en Inde ne se résume ni à l'histoire des missions, ni à celle de la colonisation, que les Indiens christianisés étaient ceux que le système social autochtone avait laissés en marge, ce qui ne les a pas conduits pour autant à renoncer à leur identité de caste, l'auteur ne révolutionne pas le domaine : il livre une synthèse des travaux les plus sérieux (dont les siens propres). Mais il fait davantage et adopte un ton plus personnel lorsqu'il écrit, de manière répétitive, qu'à toutes les raisons qui expliquent que le christianisme indien soit indigène, il faut encore en ajouter une autre, fondamentale celle-là, qui réside dans l'essence transculturelle du christianisme lui-même (Frykenberg dit plus volontiers « la foi chrétienne »). Le christianisme a toujours et partout «été capable de transcender les barrières ethniques, nationales et culturelles » (p. 454).

11 Si tel est le cas, ne manque pas de se demander le lecteur, pourquoi s'attacher avec tant de ferveur à démontrer que le christianisme a pris en Inde des formes distinctivement indiennes? Qui n'admet en effet que le christianisme en Espagne soit espagnol, en Russie russe, au Liban libanais, en France français, etc., qui n'admet qu'il ait été décliné différemment selon les Églises, les rites et les théologies catholiques, protestants, orthodoxes, etc. ? Écrirait-on un tel livre sur le caractère français du christianisme en France (question bien différente de celle du gallicanisme) ? C'est donc que la situation du christianisme en Inde est plus complexe qu'il n'y parait. Elle est plus complexe en effet, car les liens des missionnaires occidentaux avec les évangélisateurs indigènes furent foncièrement ambivalents, comme furent foncièrement ambivalents les rapports des premiers avec l'entreprise coloniale. Frykenberg ne l'ignore pas! S'il choisit de mettre l'accent sur les traits indigènes du christianisme en Inde, c'est qu'il écrit à une époque où dans le sous-continent d'aucuns opposent à l'autochtonie des hindous (83\% de la population) une vision occidentalo-centrée de l'évangélisation. Cette vision expose à d'incroyables violences la petite minorité chrétienne (entre 2,3\% - chiffres officiels - et de 6,2 à 7,3\% - selon certaines officines -, p. 464) parce qu'elle fait d'elle un implant étranger, un épiphénomène de la colonisation qui n'a plus sa place dans l'Inde indépendante. Voilà pourquoi Frykenberg se laisse parfois entraîner sur un terrain apologétique qui n'est pas celui de l'historien. 